\title{
Interações Medicamentosas em pacientes renais crônicos em hemodiálise
}

\author{
Drug Interactions in chronic kidney patients in hemodialysis \\ Interaciones Medicamentosas em pacientes renales crónicos em hemodiálisis
}

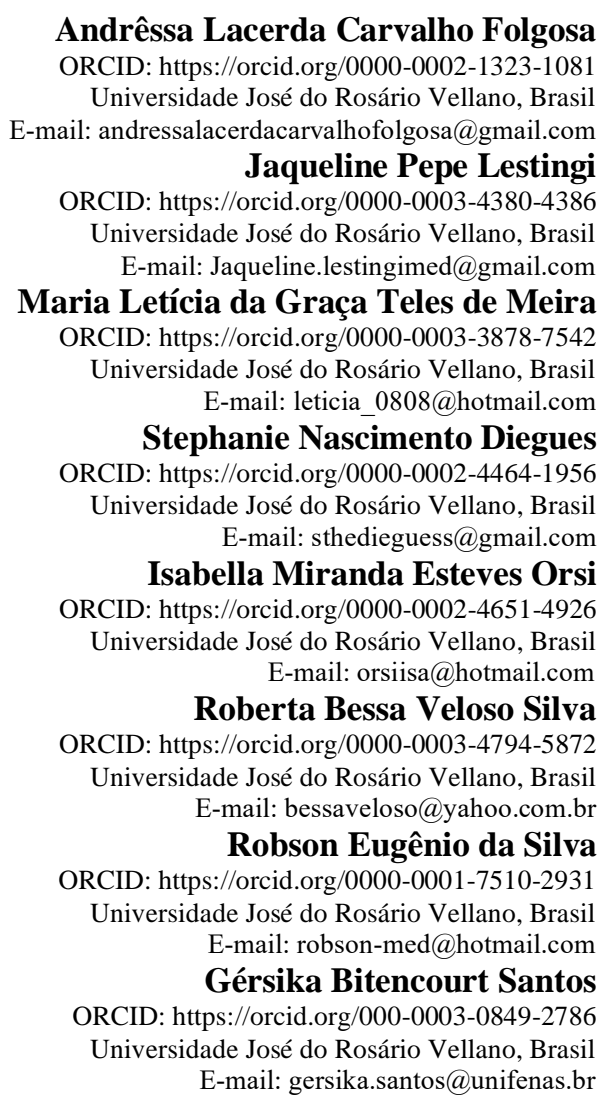

\section{Resumo}

Este trabalho tem como objetivo identificar os medicamentos utilizados com maior frequência entre os pacientes em hemodiálise e identificar possíveis interações medicamentosas entre tais fármacos. Este é um estudo transversal realizado no setor de hemodiálise de um hospital. O cruzamento entre os medicamentos foi estudado no programa Interage - Interações Medicamentosas, com a finalidade de identificar potenciais interações medicamentosas e classificá-las de acordo com a gravidade. O total de pacientes estudados foi de 45 indivíduos, destes, $47 \%$ eram homens. Os fármacos utilizados com maior frequência pelos pacientes foram Omeprazol $(22,35 \%)$, seguido pela Eritropoietina $(11,40 \%)$. Foram encontradas 113 interações medicamentosas, sendo 38 IMs de caráter leve (33,63\%), 60 IMs de caráter moderado $(53,10 \%)$ e 15 IMs de caráter grave $(13,27 \%)$. Foi considerada alta a prevalência de interações medicamentosas em pacientes usuários da hemodiálise. Os indivíduos em terapia hemodialítica carecem do uso de diversos medicamentos, logo tem suma importância a seleção cautelosa de soluções terapêuticas para o uso eficaz, racional e seguro desses medicamentos.

Palavras-chave: Interações medicamentosas; Insuficiência renal crônica; Hemodiálise.

\footnotetext{
Abstract

This study aims to identify the drugs used most frequently among patients on hemodialysis and to identify possible drug interactions between such drugs. This is a cross-sectional study carried out in the hemodialysis sector of a hospital. The crossing between drugs was studied in the Interage - Drug Interactions program, in order to identify potential drug interactions and classify them according to severity. The total number of patients studied was 45 individuals, of which $47 \%$ were men. The drugs most frequently used by patients were Omeprazole $(22.35 \%)$, followed by Erythropoietin $(11.40 \%)$. There were 113 drug interactions, 38 of which were mild MI (33.63\%), 60 moderate MI $(53.10 \%)$ and 15 severe MI (13.27\%). The prevalence of drug interactions in patients on hemodialysis was considered high. Individuals
} 
on hemodialysis therapy lack the use of several medications, so it is extremely important to carefully select therapeutic solutions for the effective, rational and safe use of these medications.

Keywords: Drug interactions; Chronic kidney disease; Hemodialysis.

\section{Resumen}

Este estudio tiene como objetivo identificar los fármacos utilizados con mayor frecuencia entre los pacientes en hemodiálisis e identificar posibles interacciones farmacológicas entre dichos fármacos. Se trata de un estudio transversal realizado en el sector de hemodiálisis de un hospital. El cruce entre fármacos se estudió en el programa Interage - Drug Interage, con el fin de identificar posibles interacciones medicamentosas y clasificarlas según su gravedad. El número total de pacientes estudiados fue de 45 individuos, de los cuales el $47 \%$ eran hombres. Los fármacos más utilizados por los pacientes fueron el omeprazol (22,35\%), seguido de la eritropoyetina (11,40\%). Hubo 113 interacciones medicamentosas, 38 de las cuales fueron MI leve (33,63\%), 60 de MI moderado $(53,10 \%)$ y 15 de MI grave $(13,27 \%)$. La prevalencia de interacciones farmacológicas en pacientes en hemodiálisis se consideró alta. Las personas en terapia de hemodiálisis carecen del uso de varios medicamentos, por lo que es extremadamente importante seleccionar cuidadosamente las soluciones terapéuticas para el uso eficaz, racional y seguro de estos medicamentos.

Palabras clave: Interacciones farmacológicas; Insuficiencia renal crónica; Hemodiálisis.

\section{Introdução}

A Doença Renal Crônica (DRC) é uma enfermidade que se resume numa perda constante e irreversível das funções fisiológicas renais (Ribeiro, et al. 2020). É uma questão de saúde pública, não só nacional como também internacional. Na fase já de DRC, pacientes com distúrbios renais sofrem um impacto desfavorável na sua expetativa e qualidade de vida (Marquito, et al. 2020). A prevalência dessa disfunção é crescente a cada dia, pois a expectativa de vida populacional cresce dia a dia mais, além dos crescentes diagnósticos de diabetes mellitus e hipertensão arterial sistêmica (HAS), os quais são as principais causas da DRC (Spavanello, et al. 2018).

Por volta de 133 mil brasileiros fazem terapia renal substitutiva (TRS) por complicações da DRC. Isso foi confirmado como mais que o dobro do que era atestado no século passado (Marquito, et al. 2020). Ademais, na fase terminal da DRC, os rins não possuem a capacidade de realização das suas funções para uma promoção da homeostase e, por razão desse distúrbio, os enfermos começam a realizar tratamentos medicamentos e, consequentemente, tratamento com hemodiálise. Este pode perdurar diariamente por toda a vida (Ribeiro, et al. 2020).

A hemodiálise consiste num procedimento em que uma máquina exerce as funções de um rim, como, por exemplo: eliminação e filtração de excesso de toxinas, minerais e líquidos (Ribeiro, et al. 2020). Todavia, apesar da utilização da Hemodiálise, ainda há uma polimedicação nos pacientes com DRC, pois eles, geralmente, possuem doenças de bases e essa polifarmácia ajuda nos seus tratamentos. Conforme um estudo realizado no estado do Rio Grande do Sul, há uma média de 7,88 diferentes medicações por paciente com DRC (Spavanello, et al. 2018). Essa grande quantidade facilita o acontecimento de Interações Medicamentosas que interferem ou podem interferir nos resultados terapêuticos e na qualidade de vida dos pacientes acometidos (Marquito, et al. 2020). Dessa forma, deve haver uma preocupação e uma observação detalhada do tratamento de cada paciente que detém a DRC.

Diante disso, este artigo tem como objetivo descrever as principais classes medicamentos frequentemente utilizados por pacientes em hemodiálise, identificando potenciais interações medicamentosas (IM) entre esses fármacos.

\section{Metodologia}

Este é um estudo do tipo transversal, de abordagem quantitativa (Pereira et al. 2018). O estudo abrange pacientes que realizam hemodiálise por um período de tempo maior que três meses, com idade igual ou superior a 18 anos, que realizam acompanhamento pelo Serviço de Nefrologia de um Hospital Universitário do Sul de Minas Gerais.

Foi realizada a coleta de dados em duas fases. Na primeira, foi consultado o prontuário de cada paciente incluído no estudo para coleta das variáveis demográficas e clínicas; e de prescrições médicas, para análise dos medicamentos que os 
pacientes utilizavam. Os medicamentos foram incluídos no estudo pela denominação comum brasileira (DCB). Na segunda fase, foi realizada uma análise das possíveis interações medicamentosas, tendo como suporte o site Interações Medicamentosas®.

As interações medicamentosas mencionadas foram agrupadas de acordo com a gravidade em leve, moderada ou grave. A categoria retratada como grave, também intitulada em outras dissertações como severa, importante ou maior, caracteriza uma potencial interação medicamentosa com ameaça à vida e/ou que demanda intervenção médica para reduzir ou prevenir reações adversas graves. A interação medicamentosa é identificada como moderada quando o resultado dela é em aumento do problema de saúde do indivíduo e/ou exige mudança no tratamento. A interação leve ou menor, pode causar efeitos clínicos mais sutis, que inclui uma elevação da frequência ou gravidade das reações adversas, e não exige uma mudança considerável na farmacoterapia.

Os dados coletados foram processados e tabulados utilizando o Microsoft Excel. As variáveis categóricas foram apresentadas como número absoluto e percentual.

Este trabalho foi aprovado pelo Comitê de Ética em Pesquisa Humana da UNIFENAS-Alfenas sob o parecer $n^{\circ}$ 1.605.648. Os pacientes que participaram da pesquisa assinaram o Termo de Consentimento Livre e Esclarecido, onde autorizam a coleta e utilização dos resultados para fins científicos.

\section{Resultados}

Dos 45, (n=45), pacientes incluídos no estudo, 24 (53,33\%) são do sexo masculino e 21 (46,67\%) do sexo feminino. Houve maior frequência de pacientes na faixa etária dos 41 aos 50 anos, 13 (28,89\%); seguido por indivíduos com 40 anos ou menos 12 (26,67\%), 11 (24,44\%) pacientes possuíam de 61 a 70 anos e $9(20,00 \%)$ dos pacientes apresentavam idade entre 51 e 60 anos. As principais causas que levaram esses pacientes a desenvolver a DRC foram demonstradas no Gráfico 1.

Gráfico 1 - Principais causas de hemodiálise.

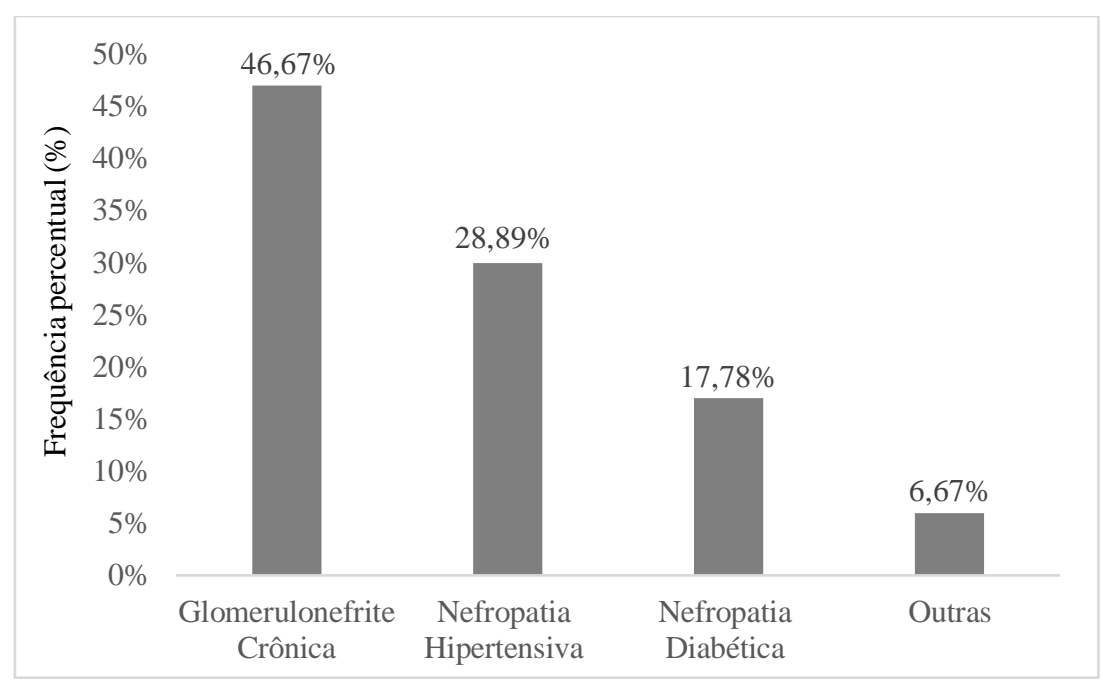

Fonte: Autores.

A Glomerulonefrite Crônica, 21 (46,67\%), a Nefropatia Hipertensiva, 13 (28,89\%) e a Nefropatia Diabética, 8 (17,78\%), entre outras, 3 (6,67\%) foram as principais causas do desenvolvimento da DRC nos indivíduos estudados.

No Gráfico 2 pode-se observar que os medicamentos mais prescritos pelo serviço prestado no tratamento da DRC foram o Omeprazol (22,35\%), seguido pela Eritropoietina (11,40\%), Furosemida (10,65\%), Sacarato de Hidróxido Férrico $(8,77 \%)$, Ácido Acetil Salicílico (AAS) (7,45\%), Carbonato de Cálcio (7,00\%) e o Alfacacifol (6,58\%). 
Gráfico 2 - Medicamentos mais prescritos pelo serviço prestado no tratamento da DRC.

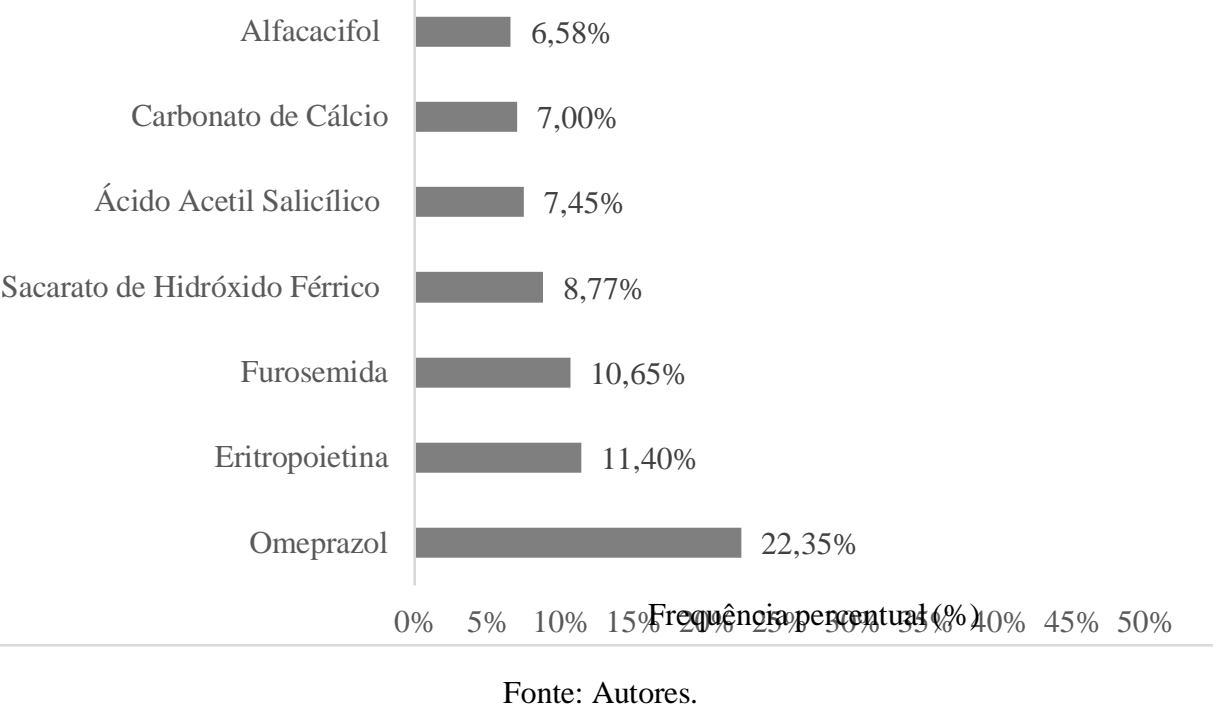

Dos prontuários analisados, $(n=45), 24(53,33 \%)$ apresentaram algum tipo de interação medicamentosa e $21(46,67 \%)$ não apresentaram essa condição. Das 113 interações medicamentosas encontradas, 38 (33,63\%) foram de caráter leve, 60 (53,10\%), moderado e 15 (13,27\%), grave. As interações medicamentosas encontradas foram listadas na Tabela 1.

Tabela 1 - Interações Medicamentosas encontradas nos prontuários dos pacientes em hemodiálise.

\begin{tabular}{|c|c|c|c|}
\hline Interações Medicamentosas & Classificação & $\mathbf{N}(\%)$ & Consequência da Interação \\
\hline Furosemida x Ácido Acetil Salicílico & Leve & $18(15,65 \%)$ & $\downarrow$ Resposta Diurética \\
\hline Ácido Acetil Salicílico x Omeprazol & Leve & $8(6,95 \%)$ & 个 pH Gástrico \\
\hline Carbonato de Cálcio x Omeprazol & Moderado & $7(6,08 \%)$ & $\downarrow$ Absoração de Cálcio \\
\hline Insulina x Ácido Acetil Salicílico & Moderado & $5(4,34 \%)$ & $\begin{array}{c}\text { 个 Concentração de Insulina } \\
\text { Basal }\end{array}$ \\
\hline Clopidogrel x Ácido acetil salicílico & Grave & $4(3,47 \%)$ & $\begin{array}{c}\uparrow \text { Hemorragias e } \\
\text { Sangramentos graves }\end{array}$ \\
\hline Carvedilol x Ácido Acetil Salicílico & Moderado & $4(3,47 \%)$ & $\downarrow$ Efeitos Anti-hipertensivos \\
\hline Furosemida $\mathrm{x}$ Enalapril & Leve & $4(3,47 \%)$ & $\begin{array}{c}\text { Inibição da Produção de } \\
\text { Angiotensina II }\end{array}$ \\
\hline Furosemida x Hidroclorotiazida & Moderado & $3(2,60 \%)$ & $\uparrow$ Diurese \\
\hline Metoprolol x Hidralazina & Moderado & $3(2,60 \%)$ & $\begin{array}{l}\text { 个 Concentrações } \\
\text { Plasmáticas }\end{array}$ \\
\hline Metoprolol x Ácido acetil salicílico & Moderado & $3(2,60 \%)$ & $\downarrow$ Efeitos Anti-hipertensivos \\
\hline Furosemida x Paracetamol & Leve & $3(2,60 \%)$ & $\downarrow$ Resposta Diurética \\
\hline Carbonato de Cálcio x Hidroclorotiazida & Moderada & $3(2,60 \%)$ & Hipercalcemia \\
\hline Levotiroxina $\mathrm{x}$ Sinvastatina & Moderado & $3(2,60 \%)$ & $\begin{array}{c}\uparrow \text { ou } \downarrow \text { Hormônio } \\
\text { Tireoideano }\end{array}$ \\
\hline Carbonato de Cálcio x Esomeprazol & Moderado & $3(2,60 \%)$ & $\downarrow$ Absoração de Cálcio \\
\hline
\end{tabular}




\begin{tabular}{|c|c|c|c|}
\hline Clopidogrel x Sinvastatina & Moderado & $2(1,73 \%)$ & $\begin{array}{l}\text { Inibição de agregacão } \\
\text { plaquetária }\end{array}$ \\
\hline Enalapril x Ácido Acetil Salicílico & Moderado & $2(1,73 \%)$ & $\downarrow$ Efeitos Vasodilatadores \\
\hline Sinvastatina $\mathrm{x}$ Diltiazem & Moderado & $2(1,73 \%)$ & $\begin{array}{l}\text { Inibição do Metabolismo de } \\
\text { HMG-CoA Redutase }\end{array}$ \\
\hline Nifedipina $x$ Omeprazol & Leve & $2(1,73 \%)$ & $\begin{array}{c}\text { 个 Concentrações } \\
\text { Plasmáticas de Nifedipina }\end{array}$ \\
\hline Propranolol x Furosemida & Leve & $2(1,73 \%)$ & $\downarrow$ Liquído Extracelular \\
\hline Propranolol $x$ Nifedipina & Moderado & $2(1,73 \%)$ & $\begin{array}{c}\text { Potencialização dos dois } \\
\text { fármacos }\end{array}$ \\
\hline Atenolol $x$ Ácido acetil salicílico (AAS) & Moderado & $2(1,73 \%)$ & $\downarrow$ Efeitos Anti-hipertensivos \\
\hline Levotiroxina $x$ Carbonato de Cálcio & Moderado & $2(1,73 \%)$ & $\downarrow$ Efeitos da Levotiroxina \\
\hline Alopurinol x Hidroclorotiazida & Grave & $1(0,86 \%)$ & $\begin{array}{c}\uparrow \text { Hipersensibilidade ao } \\
\text { Alopurinol }\end{array}$ \\
\hline Clonidina x Metoprolol & Grave & $1(0,86 \%)$ & 个 Pressão Arterial \\
\hline Clonidina $x$ Atenolol & Grave & $1(0,86 \%)$ & 个 Pressão Arterial \\
\hline Sertralina x Omeprazol & Moderado & $1(0,86 \%)$ & $\begin{array}{l}\text { 个 Efeitos Farmacológios de } \\
\text { IRS }\end{array}$ \\
\hline Diltiazem $\mathrm{x}$ Ranitidina & Moderado & $1(0,86 \%)$ & $\downarrow$ Metabolismo Hepático \\
\hline Nifedipina $x$ Diltiazem & Leve & $1(0,86 \%)$ & $\begin{array}{c}\text { 个 Concentrações } \\
\text { Plasmáticas de Nifedipina }\end{array}$ \\
\hline Atenolol $\mathrm{x}$ Carbonato de Cálcio & Moderado & $1(0,86 \%)$ & $\begin{array}{c}\downarrow \text { Efeito Farmacológico de } \\
\text { Atenolol }\end{array}$ \\
\hline Atenolol $x$ Nifedipina & Moderado & $1(0,86 \%)$ & $\begin{array}{l}\text { Potencialização dos dois } \\
\text { farmácos }\end{array}$ \\
\hline Sinvastatina $\mathrm{x}$ Eritromicina & Grave & $1(0,86 \%)$ & Severa Miopatia \\
\hline Propanolol x Hidralazina & Moderado & $1(0,86 \%)$ & $\begin{array}{l}\text { 个 Concentrações } \\
\text { Plasmáticas }\end{array}$ \\
\hline Clopidogrel x Pantoprazol & Grave & $1(0,86 \%)$ & $\downarrow$ Atividade Anti-plaquetária \\
\hline Propanolol x Ácido Acetil Salicílico & Moderado & $1(0,86 \%)$ & $\downarrow$ Efeitos Anti-hipertensivos \\
\hline Varfarina x Propanolol & Moderado & $1(0,86 \%)$ & 个 Do Efeito da Varfarina \\
\hline Varfarina x Ácido Acetil Salicílico & Grave & $1(0,86 \%)$ & $\begin{array}{l}\text { 个 Atividade de } \\
\text { Anticoagulantes }\end{array}$ \\
\hline Levotiroxina $x$ Sulfato Ferroso & Moderado & $1(0,86 \%)$ & $\downarrow$ Efeitos da Levotiroxina \\
\hline Prednisona X Ácido Acetil Salicílico & Moderado & $1(0,86 \%)$ & $\begin{array}{l}\downarrow \text { Diminuição dos níveis } \\
\text { séricos de Salicilato }\end{array}$ \\
\hline Clopídrogel x Esomeprazol & Grave & $1(0,86 \%)$ & $\begin{array}{l}\text { Interferência no processo } \\
\text { metabólico do Clopidrogel }\end{array}$ \\
\hline Metoprolol x Nifedipina & Moderado & $1(0,86 \%)$ & $\begin{array}{l}\text { Potencialização dos dois } \\
\text { farmácos }\end{array}$ \\
\hline Diclofenaco $\times$ Sertralina & Grave & $1(0,86 \%)$ & $\begin{array}{c}\uparrow \text { Risco de Hemorragia } \\
\text { Digestiva }\end{array}$ \\
\hline
\end{tabular}


Research, Society and Development, v. 10, n. 2, e44510212789, 2021

(CC BY 4.0) | ISSN 2525-3409 | DOI: http://dx.doi.org/10.33448/rsd-v10i2.12789

\begin{tabular}{|c|c|c|c|}
\hline Digoxina x Amiodarona & Grave & $1(0,86 \%)$ & $\begin{array}{c}\text { 个 Concentrações } \\
\text { Plasmáticas de Digoxina }\end{array}$ \\
\hline Digoxina x Sertralina & Moderado & $1(0,86 \%)$ & $\begin{array}{c}\text { 个 Concentrações } \\
\text { Plasmáticas de Digoxina }\end{array}$ \\
\hline Metoprolol x Amiodarona & Moderado & $1(0,86 \%)$ & $\uparrow$ dos Efeitos do Metoprolol \\
\hline Metoprolol x Sertralina & Moderado & $1(0,86 \%)$ & $\begin{array}{c}\text { 个 dos efeitos dos Beta- } \\
\text { bloqueadores }\end{array}$ \\
\hline Varfarina x Amiodarona & Grave & $1(0,86 \%)$ & Efeito hiprotrombinêmico \\
\hline Varfarina x Diclofenaco & Grave & $1(0,86 \%)$ & $\begin{array}{l}\text { 个 Atividade de } \\
\text { Anticoagulantes }\end{array}$ \\
\hline Varfarina x Paracetamol & Moderado & $1(0,86 \%)$ & 个 Efeito Antitrombótico \\
\hline Varfarina x Prednisona & Moderado & $1(0,86 \%)$ & $\begin{array}{l}\text { Alteração do efeito de } \\
\text { anticoagulantes }\end{array}$ \\
\hline Varfarina $\times$ Sertralina & Moderado & $1(0,86 \%)$ & $\begin{array}{c}\text { 个 dos Efeitos } \\
\text { Anticoagulantes }\end{array}$ \\
\hline
\end{tabular}

Fonte: Autores.

Verificou-se que das 38 (33,04\%) interações medicamentosas de caráter leve, 18 (47,37\%) usaram furosemida e ácido acetil salicílico; 8 (21,06\%), ácido acetil salicílico e omeprazol; 4 (10,53\%), furosemida e enalapril; 3 (7,89\%), furosemida e paracetamol; 2 (5,26\%), nifedipina e omeprazol; 2 (5,26\%), propranolol e furosemida; e 1 (2,63\%), nifedipina e diltiazem. As consequências destas interações foram, respectivamente, a baixa resposta diurética, alto $\mathrm{pH}$ gástrico, inibição da produção de angiotensina II, baixa resposta diurética, altas concentrações plasmáticas de nifedipina, baixo liquído extracelular, altas concentrações plasmáticas de nifedipina.

Em se tratando das $60(53,10 \%)$ interações medicamentosas de grau moderado, sendo cada uma delas correspondente a $1(1,67 \%)$ paciente, verificou-se a sertralina e omeprazol, diltiazem e ranitidina, atenolol e carbonato de cálcio, atenolol e nifedipina, propanolol e hidralazina, propranolol e ácido acetil salicílico, varfarina e propranolol, levotiroxina e sulfato ferroso, prednisona e ácido acetil salicílico, metoprolol e nifedipina, digoxina e sertralina, metoprolol e amiodarona, metoprolol e sertralina, varfarina e paracetamol, varfarina e paracetamol, varfarina e sertralina.

Como consequências destas interações verificou-se, respectivamente, alta dos efeitos farmacológicos de IRS, potencialização dos dois fármacos, altas concentrações plasmáticas, altos efeitos anti-hipertensivos, alto efeito da varfarina, baixos efeitos da levotiroxina, diminuição dos níveis séricos de salicilato, potencialização dos dois fármacos, altas concentrações plasmáticas de digoxina, alta dos efeitos do metoprolol, betabloqueadores, antitrombótico, alteração do efeito de anticoagulantes e alta dos efeitos anticoagulantes.

Com uma frequência de 2 (3,33\%) pacientes enquadrados no grau moderado, pode-se observar como consequências das seguintes interações, clopidogrel e sinvastatina, enalapril e ácido acetil salić́lico, sinvastatina e diltiazem, propranolol e nifedipina, atenolol e ácido acetil salicílico, e levotiroxina e carbonato de cálcio, respectivamente, a inibição de agregação plaquetária, baixos efeitos vasodilatadores, inibição do metabolismo de HMG-CoA Redutase, potencialização dos dois fármacos, baixo efeitos anti-hipertensivos e baixos efeitos da levotiroxina.

As interações medicamentosas observadas em $3(5,00 \%)$ pacientes foram a furosemida e hidroclorotiazida, metoprolol e hidralazina, metoprolol e ácido acetil salicílico, carbonato de cálcio e hidroclorotiazida, levotiroxina e sinvastatina, carbonato de cálcio e esomeprazol, ocasionando à alta diurese, altas concentrações plasmáticas, baixos efeitos anti-hipertensivos, hipercalcemia, alteração no hormônio tireoideano e baixa absorção de cálcio. Em 4 (6,67\%) pacientes observou-se o uso de 
carvedilol e ácido acetil salicílico e, consequentemente, baixos efeitos anti-hipertensivos. Com uma frequência de 5 (8,33\%) pacientes verificou-se a interação entre a insulina e o ácido acetil salicílico, resultando em altas concentrações de insulina basal. A interação entre o carbonato de cálcio e omeprazol, foi observada em 7 (11,67\%) pacientes, tendo como consequência a baixa absorção de cálcio.

Ao considerar as interações de caráter grave em 15 (13,27\%) pacientes, àquelas com uma frequência de $1(6,67 \%)$ paciente, observou-se a utilização de alopurinol e hidroclorotiazida, clonidina e metoprolol, clonidina e atenolol, sinvastatina e eritromicina, clopidogrel e pantoprazol, varfarina e ácido acetil salicílico, clopidrogel e esomeprazol, diclofenaco e sertralina, digoxina e amiodarona, varfarina e amiodarona, varfarina e diclofenaco. As consequências foram alta hipersensibilidade ao alopurinol, alta pressão arterial tanto para clonidina e metoprolol quanto para clonidina e atenolol, severa miopatia, baixa atividade antiplaquetária, alta atividade anticoagulante, alta atividade de anticoagulantes, interferência no processo metabólico do clopidrogel, alto risco de hemorragia digestiva, altas concentrações plasmáticas de digoxina, efeito hiprotrombinêmico, alta atividade de anticoagulantes, efeito hiprotrombinêmico e alta atividade anticoagulante. Em 4 (26,67\%) pacientes pode-se observar o uso de clopidogrel e ácido acetil salicílico, acarretando altas hemorragias e sangramentos graves.

Observou-se que houveram interações medicamentosas mais frequentes em pacientes com DRC (Gráfico 3).

Gráfico 3 - Interações medicamentos mais frequentes em pacientes com DRC.

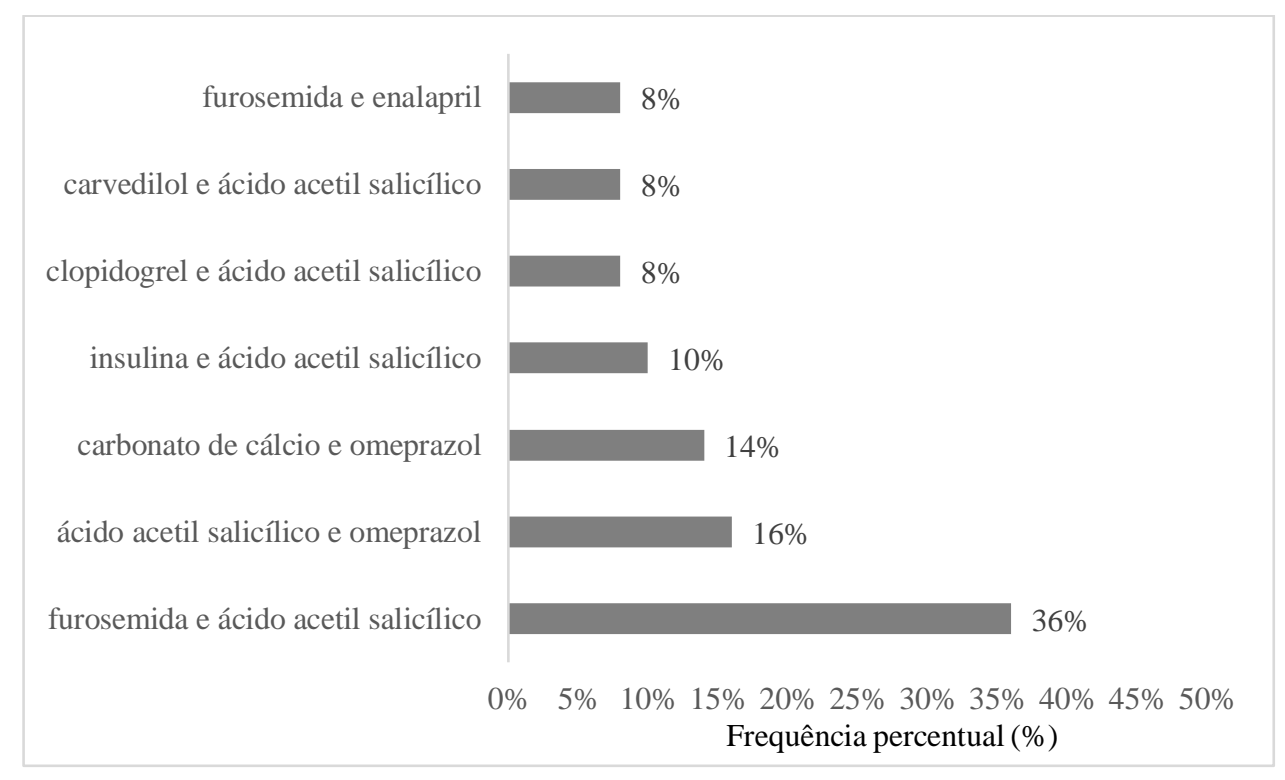

Fonte: Autores.

Das interações mais frequentes destacam-se 8 (16,00\%) entre o ácido acetil salicílico e omeprazol; 7 (14,00\%) entre carbonato de cálcio e omeprazol, 5 (10,00\%) entre insulina e ácido acetil salić́lico; 4 (8,00\%) entre clopidogrel e ácido acetil salicílico, sendo esta última de caráter grave; 4 (8,00\%) entre carvedilol e ácido acetil salicílico; 4 (8,00\%) entre furosemida e enalapril, e 18 (36,00\%) entre furosemida e ácido acetil salicílico.

\section{Discussão}

Neste estudo foi mostrada uma prevalência de pacientes do sexo masculino. Em contrapartida, outros trabalhos com outras populações mostraram uma singela prevalência feminina (Marquito, et al. 2020) (Sgnaolim, et al. 2014) (Spavanello, et al. 2018). Em relação à idade, foi mostrada uma predominância dos pacientes na faixa etária dos 41 aos 50 anos os que mais 
realizavam hemodiálise. Spavanello et al. 2018 mostrou a faixa etária mais acometida foi a população entre 45 a 65 anos. Isso foi análogo aos dados de 90356 pacientes da Base Nacional em Terapias Renais Substitutivas.

As interações medicamentosas foram classificadas em leves, moderadas e graves, neste estudo. Foi notado que as interações moderadas foram as mais encontradas, assim como em dois estudos realizados de modo separado no estado do Rio Grande do Sul (Sgnaolim, et al. 2014; Spavanello, et al. 2018).

O presente estudo obteve resultados que comprovaram que a nefropatia diabética foi predominante nas doenças de base que causaram essa condição crônica na população estudada. Outros trabalhos também mostraram a relevância das complicações da diabetes mellitus no desenvolvimento da DRC. Contudo, foi observado nestes estudos que a associação entre Nefropatia Diabética e a Nefropatia Hipertensiva são condições importantes para o desenvolvimento da DRC (Ribeiro, et al. 2020; Sgnaolim, et al. 2014).

Os medicamentos mais utilizados pelos pacientes deste estudo foram Omeprazol, Eritropoietina, Furosemida, Sacarato de Hidróxido Férrico, Ácido Acetil Salicílico (AAS), Carbonato de Cálcio e Alfacacifol. Existiu uma modesta distinção nas drogas mais consumidas em um estudo realizado em Juiz de Fora - MG, que afirma que os medicamentos mais utilizados pela população que realiza hemodiálise em ordem decrescente foram: Losartana Potássica, Sinvastatina, Furosemida, AAS, Bensilato de Anlopidino, Omeprazol, Insulina Humana NPH (Marquito, et al. 2020). Segundo SGNAOLIM et al., 2014 houve uma maior semelhança nos medicamentos mais utilizados no presente estudo, porém tendo uma diferença nas ordens. Os medicamentos mais utilizados pela população estudada por Sganaolim foram Carbonato de Cálcio, Eritropoietina, Citrato de Sódio, AAS, Omeprazol, Calcitriol e Sinvastatina, nesta ordem.

Com a grande quantidade de medicamentos ingeridos para enfermos com DRC, a probabilidade de IMs se torna cada vez maior (Ribeiro, et al. 2020). Neste estudo a interação de maior frequência foi a associação entre a Furosemida e o AAS, que pode reduzir o efeito diurético e vasodilatador da Furosemida, tendo o risco de ototoxicidade aumentada com altas doses de salicilato por diuréticos como o Furosemida. E outro estudo, foi concluído que a associação entre o Anlodipino e a Sinvastatina foi a mais frequente. Essa IM pode causar uma ampliação nas concentrações séricas da sinvastatina e no risco de miopatia com inclusão da rabdomiólise (Spavanello, et al. 2018).

Os responsáveis pelas prescrições desses medicamentos devem estar atentos às suas associações para que possam apontar a presença de potenciais IM. Outros métodos para se ter uma precaução de futuras IM é possuir um domínio dos fatores de risco correlacionados à IM, inclusive a DRC, além da noção de que cada medicamento acrescentado a um processo terapêutico eleva, significativamente, a ameaça de IM (Gagne, Maio \& Rabinowitz, 2008).

\section{Conclusão}

As prováveis interações medicamentosas em pacientes no tratamento de hemodiálise obtiveram uma prevalência alta. A população com doença renal crônica, devido às várias condições mórbidas, utiliza vários medicamentos concomitantemente. Sendo assim, este trabalho mostra a importância do profissional da área de saúde durante a análise dos medicamentos utilizados pelos pacientes com o intuito melhorar a resposta terapêutica e reduzir os danos ocasionados por tais interações. Salienta-se a necessidade de ser estudado mais detalhadamente em outras populações polimedicadas, visando resultados adicionais que possam vir a colaborar com nossos achados.

\section{Referências}

Bastos, M. G., Bregman, R., \& Kirsztajn, G. M. (2010). Doença renal crônica: frequente e grave, mas também prevenível e tratá vel. Revista da Associação Médica Brasileira, 56(2), 248-253. 
Bastos, M. G., \& Kirsztajn, G. M. (2011). Doença renal crônica: importância do diagnóstico precoce, encaminhamento imediato e abordagem interdisciplinar estruturada para melhora do desfecho em pacientes ainda não submetidos à diálise. Brazilian Journal of Nephrology, 33(1), 93-108.

Corrêa, J. C., \& Corrêa, V. L. F. (1998). Antibióticos no dia-a-dia. Livraria Rubio.

Costa, G. R., \& Nascimento, P. S. (2013). Análise de prescrições dos pacientes submetidos à hemodiálise e avaliação de possív eis interações medicamentosas. Trabalho de Conclusão de Curso apresentado ao curso de farmácia da Faculdade de Ceres, 10-40.

Doubova, S. V., Reyes-Morales, H., del Pilar Torres-Arreola, L., \& Suárez-Ortega, M. (2007). Potential drug-drug and drug-disease interactions in prescriptions for ambulatory patients over 50 years of age in family medicine clinics in Mexico City. BMC health services research, 7(1), 1-8.

Gagne, J. J., Maio, V., \& Rabinowitz, C. (2008). Prevalence and predictors of potential drug-drug interactions in Regione Emilia-Romagna, Italy. Journal of clinical pharmacy and therapeutics, 33(2), 141-151.

Hammes, J. A., Pfuetzenreiter, F., Silveira, F. D., Koenig, Á., \& Westphal, G. A. (2008). Prevalência de potenciais interações medicamentosas droga-droga em unidades de terapia intensiva. Revista Brasileira de Terapia Intensiva, 20(4), 349-354.

Hoefler, R. (2005). Interações medicamentosas. Secretaria de Ciência, Tecnologia e Insumos Estratégicos/MS-FTN, 1, 1-4.

Kirsztajn, G. M., Bastos, M. G., \& Andriolo, A. (2011). Dia Mundial do Rim 2011 Proteinúria e creatinina sérica: testes essenciais para diagnóstico de doença renal crônica. Jornal Brasileiro de Patologia e Medicina Laboratorial, 47(2), 100-103.

Mannheimer, B., Wettermark, B., Lundberg, M., Pettersson, H., Von Bahr, C., \& Eliasson, E. (2010). Nationwide drug-dispensing data reveal important differences in adherence to drug label recommendations on CYP2D6-dependent drug interactions. British journal of clinical pharmacology, $69(4), 411-417$.

Marquito, A. B., Fernandes, N. M. D. S., Colugnati, F. A. B., \& Paula, R. B. D. (2014). Interações medicamentosas potenciais em pacientes com doença renal crônica. Brazilian Journal of Nephrology, 36(1), 26-34.

Marquito, A. B., Pinheiro, H. S., Fernandes, N. M. D. S., \& Paula, R. B. D. (2020). Avaliação da farmacoterapia na doença renal crônica: valid ação do instrumento PAIR para uso no Brasil. Brazilian Journal of Nephrology, (AHEAD).

Martins, M. R. I., \& Cesarino, C. B. (2005). Qualidade de vida de pessoas com doença renal crônica em tratamento hemodialítico. Revista Latino-Americana de Enfermagem, 13(5), 670-676

Moore, K. L. (2013). Anatomia orientada para a clínica. In Anatomia orientada para a clínica (pp. 1104-1104).

Moura, C., Prado, N., \& Acurcio, F. (2011). Potential drug-drug interactions associated with prolonged stays in the intensive care unit. Clinical Drug Investigation, 31(5), 309-316

Pereira, A. S. et al. (2018). Metodologia da pesquisa científica. UFSM. https://repositorio.ufsm.br/bitstream/handle/1/15824/Lic_Computacao_MetodologiaPesquisa-Cientifica.pdf?sequence $=1$.

Pirmohamed, M. (2010). Drug-drug interactions and adverse drug reactions: separating the wheat from the chaff. Wiener klinische Wochenschrift, 122(3), 6264.

Ribeiro, W. A., de Oliveira Jorge, B., \& de Sena Queiroz, R. (2020). Repercussões da hemodiálise no paciente com doença renal crônica: uma revisão da literatura. Revista Pró-UniverSUS, 11(1), 88-97.

Sgnaolin, V., Sgnaoli, V., Engroff, P., DeCarli, G. A., \& Figueiredo, A. E. P. L. (2014). Avaliação dos medicamentos utilizados e possíveis interações medicamentosas em doentes renais crônicos. Scientia Medica (PUCRS. Impresso).

Spanevello, S., Locatelli, C., Bandeira, V. A. C., \& de Fátima Colet, C. (2018). Interações medicamentosas, reações adversas e ajuste de dose de medicamentos utilizados por pacientes em hemodiálise. Saúde (Santa Maria), 3(44)

Tortora, G. J., \& Derrickson, B. (2016). Corpo Humano-: Fundamentos de Anatomia e Fisiologia. Artmed Editora. 